\title{
Atypical Kawasaki in a 15-Month-0ld Infant: Diagnosis Is Not Always Easy
}

\author{
M. Ech-Chebab, M. Rkain, A. Babakhouya, Y. Sbiaa, A. Ghannam, A. Elouali, N. Benajiba \\ Department of Pediatrics, Mohammed VI University Hospital Center of Oujda, Oujda, Morocco \\ Email: med.ech.chebab@gmail.com
}

How to cite this paper: Ech-Chebab, M., Rkain, M., Babakhouya, A., Sbiaa, Y., Ghannam, A., Elouali, A. and Benajiba, N. (2020) Atypical Kawasaki in a 15-Month-Old Infant: Diagnosis Is Not Always Easy. World Journal of Cardiovascular Diseases, 10, 483-487. https://doi.org/10.4236/wjcd.2020.107048

Received: June 12, 2020

Accepted: July 24, 2020

Published: July 27, 2020

Copyright $\odot 2020$ by author(s) and Scientific Research Publishing Inc. This work is licensed under the Creative Commons Attribution International License (CC BY 4.0).

http://creativecommons.org/licenses/by/4.0/

\begin{abstract}
Introduction: Kawasaki disease (KD) is an acute systemic febrile vasculitis in young children and infants, which affects medium and small caliber vessels. Case report: A 15-month-old male patient who initially consulted for left cervical lymphadenopathy against a background of fever. The diagnosis of an adenophlegmon has been suspected. Outpatient treatment was administered. The course was marked by the persistence of fever and regression of lymphadenopathy. Clinical examination during the check found cheilitis and peeling of the perineum with biological inflammatory syndrome. The patient received aspirin anti-inflammatory dose and perfusion of immunoglobulins with good progress. Conclusion: In the case of an adenopathy in infants associated with prolonged fever, one should always be wary of a Kawasaki even in the absence of other clinical criteria.
\end{abstract}

\section{Keywords}

Kawasaki, Vasculitis, Lymphadenopathy, Fever, Aneurysm

\section{Introduction}

Kawasaki disease $(\mathrm{KD})$ is an acute systemic febrile vasculitis in young children and infants, which affects medium and small caliber vessels [1]. It is the leading cause of acquired heart disease in developed nations and is slowly bypassing rheumatic heart disease in developing countries [2] [3]. The majority (80\%) of patients are less than 5 years old, with a peak incidence around 12 months of age [4]. The clinical criteria of Kawasaki can guide the diagnosis in typical cases, but sometimes the diagnosis is not so easy, we report a case of atypical Kawasaki in a 15-month-old infant. 


\section{Case Report}

Our patient is a 15-month-old male with no significant history, who initially consulted ( 6 days before admission) for left cervical lymphadenopathy with inflammatory signs opposite measuring $6 \mathrm{~cm}$ in its major axis evolving in a context of fever. The diagnosis of an adenophlegmon was suspected, a cervical CT was carried out showing a fluid formation in the process of superinfection without image of abscess. Outpatient treatment was administered (antibiotic, antipyretic, corticosteroid). The evolution was marked by the persistence of fever $\left(38.8^{\circ}-40^{\circ}\right)$ and regression of lymphadenopathy. Clinical examination during the control found cheilitis and peeling of the perineum. The biological assessment found a sedimentation rate accelerated to: $107 \mathrm{~s}$ (1st hour), CRP rose to 70 $\mathrm{mg} / \mathrm{l}$, a thrombocytosis to $800,000 / \mathrm{mm}^{3}$. The echocardiography showed a left coronary dilation of $4 \mathrm{~mm}$. The patient received anti inflammatory dose aspirin and immunoglobulin perfusion with good clinical and biological progress.

\section{Discussion}

In more than three quarters of reported cases, the Kawasaki desease affects children between 6 months and 5 years old [5], with a maximum around the age of 2 years [6]. It affects boys more often ( 3 boys for 2 girls). The disease is rare before the age of 3 months, which suggests protection by antibodies of maternal origin, and it is very rare in adolescents and adults [7]. The etiology of KD is not known. The presence of familial clusters and increased incidence in Asian populations indicate the presence of a genetic component [8] [9]. Kawasaki disease often begins with a high and persistent fever that is not very responsive to normal treatment with paracetamol (acetaminophen) or ibuprofen [10] [11]. In addition to fever, there are 5 other criteria, the diagnosis of Kawasaki disease can be retained if the fever is associated with at least 4 of these 5 criteria [12]. Further symptoms include generalized polymorphic exanthema (>90\%), palmoplantar erythema (80\%), symmetric non purulent conjunctivitis $(80 \%-90 \%)$, usually unilateral cervical lymphadenopathy $(>1.5 \mathrm{~cm} ; 50 \%)$, and mucosal enanthema with red and/or chapped lips (80\% - 90\%) [13]. In this observation, the diagnosis of Kawasaki disease was not immediately mentioned. The initial presentation with isolated cervical adenitis is however frequent in the small infant [14]. Cervical lymphadenopathy in the context of acute fever is mistakenly taken as infectious in front of the radiological image of abscess "Figure 1" given the frequency of it in emergency consultations, which often leaves out the diagnosis of a Kawasaki disease in its acute phase; the persistence of fever despite Antibiotic and antipyretic should suggest Kawasaki disease. Our patient did not present a typical clinical picture of Kawasaki initially, but later during a control consultation he presented a cheilitis with perineal scaling. In front of all these criteria, the diagnosis of a Kawasaki disease was strongly suspected. An echocardiography was requested to look for coronary artery disease and to propose therapeutic management, which showed a left coronary dilation of $4 \mathrm{~mm}$. In the 


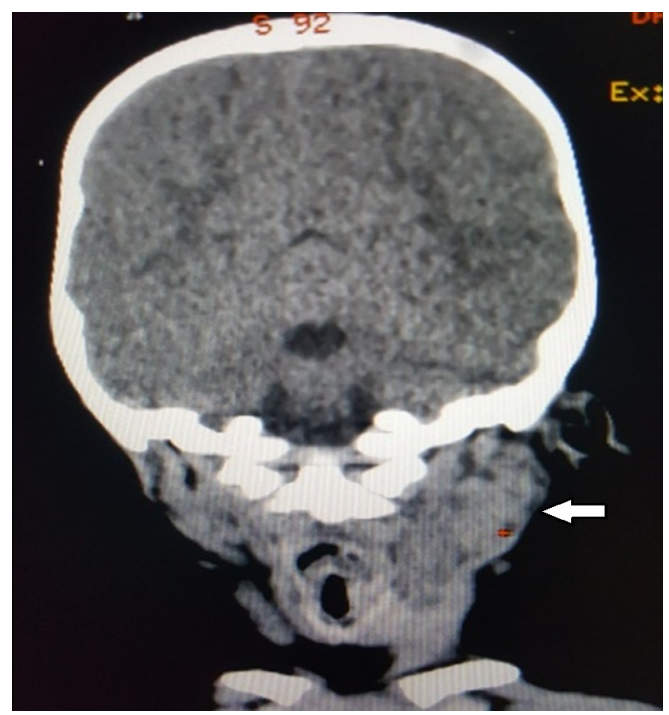

Figure 1. Axial CT section showing the cervical lymphadenopathy.

atypical form of Kawasaki disease the diagnosis can be considered confirmed when coronary artery aneurysms are identified in such patients by echocardiography [15]. Our patient received anti-inflammatory aspirin and an immunoglobulin infusion (1g per kg per day for 2 days). According to Brian W. McCrindle et al. 2017; immunoglobulins are offered in such cases, even if not administered early during illness. The course of Kawasaki disease is most often favorable in the absence of coronary complications. Relapses are possible but rare. The dimensions of the coronary aneurysm seem to be an important prognostic factor [16]. Careful initial management of evolving coronary artery abnormalities is essential, necessitating an increased frequency of assessments and escalation of thromboprophylaxis. Risk stratification for long-term management is based primarily on maximal coronary artery luminal dimensions, normalized as $\mathrm{Z}$ scores, and is calibrated to both past and current involvement. Patients with aneurysms require life-long and uninterrupted cardiology follow-up [15].

The regression of aneurysms occurs in $60 \%$ of cases within 2 years. Among the long-term sequelae, recent data suggest a risk of coronary arteriosclerosis in adulthood, as well as morphological (intestinal thickening) and functional (reduced contractility) abnormalities in the coronary arteries [17]. In our patient, we noted a gradual regression of the aneurysm after 1 year of evolution; he is currently under radiological control every 3 to 6 months without any other complication.

\section{Conclusions}

When faced with lymphadenopathy in infants associated with prolonged fever, one should always be wary of Kawasaki disease even in the absence of other clinical criteria.

The diagnosis is urgent in order to take charge of the infant early before the installation of complications, which can be harmful, in particular, coronary ar- 
tery disease.

\section{Conflicts of Interest}

The authors declare no conflicts of interest regarding the publication of this paper.

\section{References}

[1] Newburger, J.W., Takahashi, M., Gerber, M.A., et al. (2004) Diagnosis, Treatment, and Long-Term Management of Kawasaki Disease: A Statement for Health Professionals from the Committee on Rheumatic Fever, Endocarditis, and Kawasaki Disease, Council on Cardiovascular Disease in the Young, American Heart Association. Circulation, 110, 2747-2771. https://doi.org/10.1161/01.CIR.0000145143.19711.78

[2] Saguil, A., Fargo, M. and Grogan, S. (2015) Diagnosis and Management of Kawasaki Disease. American Family Physician, 91, 365-371.

[3] Vervoort, D., Donné, M. and Van Gysel, D. (2018) Pitfalls in the Diagnosis and Management of Kawasaki Disease: An Update for the Pediatric Dermatologist. Pediatric Dermatology, 35, 743-747. https://doi.org/10.1111/pde.13620

[4] Pannaraj, P.S., Turner, C.L., Bastian, J.F. and Burns, J.C. (2004) Failure to Diagnose Kawasaki Disease at the Extremes of the Pediatric Age Range. The Pediatric Infectious Disease Journal, 23, 789-791. https://doi.org/10.1097/01.inf.0000134312.39744.a4

[5] Harnden, A., Takahashi, M. and Burgner, D. (2009) Kawasaki Disease. BMJ, 338, b1514. https://doi.org/10.1136/bmj.b1514

[6] Uehara, R. and Belay, E.D. (2012) Epidemiology of Kawasaki Disease in Asia, Europe, and the United States. Journal of Epidemiology, 22, 79-85. https://doi.org/10.2188/jea.JE20110131

[7] Brocco, C. (2019) Kawasaki infantile: Quand y penser? La Revue du Praticien-Médecine Générale, 33, 168-169.

[8] Nakamura, Y., Yashiro, M., Uehara, R., Sadakane, A., Tsuboi, S., Aoyama, Y., et al. (2012) Epidemiologic Features of Kawasaki Disease in Japan: Results of the 2009-2010 Nationwide Survey. Journal of Epidemiology, 22, 216-221. https://doi.org/10.2188/jea.JE20110126

[9] Dergun, M., Kao, A., Hauger, S.B., Newburger, J.W. and Burns, J.C. (2005) Familial Occurrence of Kawasaki Syndrome in North America. Archives of Pediatrics and Adolescent Medicine, 159, 876-881. https://doi.org/10.1001/archpedi.159.9.876

[10] Kawasaki, T. (1967) Acute Febrile Mucocutaneous Syndrome with Lymphoid Involvement with Specific Desquamation of the Fingers and Toes in Children. Arerugi, 16, 178-222.

[11] Rowley, A.H. and Shulman, S.T. (1998) Kawasaki Syndrome. Clinical Microbiology Reviews, 11, 405-414. https://doi.org/10.1128/CMR.11.3.405

[12] Cimaz, R. and Lega, J.-C. (2007) La maladie de Kawasaki. Encyclopédie Orphanet.

[13] Burns, J.C., Mason, W.H., Glode, M.P., Shulman, S.T., Melish, M.E., Meissner, C., et al. (1991) Clinical and Epidemiologic Characteristics of Patients Referred for Evaluation of Possible Kawasaki Disease. United States Multicenter Kawasaki Disease Study Group. The Journal of Pediatrics, 118, 680-686.

https://doi.org/10.1016/S0022-3476(05)80026-5

[14] Rosenfeld, E.A., Corydon, K.E. and Shulman, S.T. (1995) Kawasaki Disease in In- 
fants Less than One Year of Age. The Journal of Pediatrics, 126, 524-529. https://doi.org/10.1016/S0022-3476(95)70344-6

[15] McCrindle, B.W., et al. (2017) Diagnosis, Treatment, and Long-Term Management of Kawasaki Disease a Scientific Statement for Health Professionals from the American Heart Association. Circulation, 135, e927-e999.

[16] Levy, D.M., Silverman, D., Massicotte, P., et al. (2005) Longterm Outcomes in Patients with Giant Aneurysms Secondary to Kawasaki Disease. The Journal of Rheumatology, 32, 928-934.

[17] Iemura, M., Ishii, M., Sugimura, T., Akagi, T. and Kato, H. (2000) Long Term Consequences of Regressed Coronary Aneurysms after Kawasaki Disease: Vascular Wall Morphology and Function. Heart, 83, 307-311.

https://doi.org/10.1136/heart.83.3.307 